\title{
Editorial
}

\section{Psychosocial risks and work-related stress}

\section{Dra. Valentina Forastieri}

Senior Specialist on Occupational Health. Programme on Health Promotion and Well-being. International Labour Office.

\section{Correspondencia}

Dra. Valentina Forastieri

Specialist on Occupational Health

Programme on Health Promotion and Well-being International Labour Office

Correo electrónico: forastieri@ilo.org 
The pace of modern life has increased considerably as compared with 20 years ago. Globalization and technological progress have transformed the way we work across the world. Consequently we are facing:

- New and more complex technologies in all fields, and in particular in communications;

- New forms of work organization, including work intensification and working time arrangements, the fragmentation of production processes ,out-sourcing and organizational restructuring;

- New forms of employment, with prevalence of temporary, part-time and home-based work;

- Job insecurity, higher demands and precarious employment which have intensified in developed countries with the crisis and recession;

- Demographic and political changes in the workforce composition due to migration, the ageing of the workforce, lack of access to employment of young workers and an increasing number of workers in the informal economy.(1)

As a consequence, psychosocial hazards and risks have emerged. According to the ILO definition from 1986, "psychosocial factors at work refer to interactions between and among work environment, job content, work organisation and workers' capacities, needs, culture, personal extra-job considerations that may, through perceptions and experience, influence health, work performance and job satisfaction". It is the interaction of all these variables that is recognised as having a potentially hazardous effect on workers' health (2). Stress researchers have identified nine categories under which psychosocial risks can be grouped: job content; workload and work pace; working hours; participation and control; career development, status and pay; role in the organization; interpersonal relations; organizational culture and home-work interface (3). Work-related stress is the harmful physical and emotional response caused by an imbalance between the perceived demands and the perceived resources and abilities of a person to cope with those demands. This occurs when the demands of the job do not match or exceed the capabilities, resources, or needs of the worker and when the knowledge and abilities to cope of an individual worker or of a group are not matched with the expectations of the organizational culture of an enterprise. As psychosocial risks are determined by work organization and labour relations, most of the causes of work-related stress are associated with how work content is designed and how work is organized.

In the light of the evidence from previous crises, the recent economic crisis and current recession in developed countries, it is expected that the number of workplace accidents and ill-health will rise due to enterprises' restructuring and unemployment. The evidence shows that working conditions and quality are put at risk due to restructuring. Organizational changes in this period may lead to a decrease in the management of traditional hazards and risks, under the argument of a necessary reduction in costs. As occupational safety and health is still perceived by many enterprises as a cost rather than an investment, some companies may be tempted to ignore occupational safety and health (OSH) standards. The decrease in public spending will also compromise the capacities to deliver of labour inspectorates and other OSH services. Furthermore, many enterprises lack the means to deal with emerging psychosocial risks or are unaware of their underlying causes.

In the new working context, stress can be triggered by workplace reorganization through perceived lack of control over work, job insecurity and changes in working time patterns or job demands due to the intensification of work or workers being required to be more flexible and to learn faster in order to perform new tasks. Organizational change causes uncertainty and antagonisms at all levels of the organization and society. It is likely to be associated with an increased incidence of psychosocial risks at work causing workrelated stress, anxiety depression and fatigue which can then lead to accidents and diseases, reduce performance and productivity, and affect working relations(4). Furthermore, with organizational changes and restructuring (downsizing, out-sourcing, subcontracting) workers have to deal with the fear of losing their jobs, reduced opportunities for advancement and massive layoffs. Such factors are likely to be more significant as employment becomes more precarious for some, and workloads and working hours often increase for those remaining in employment causing stress and other health disorders. Consequently in recent years, work-related stress and burnout have escalated; coping behaviours to deal with stress, such as
(1) ILO, 2009.
(2) ILO, 1986.
(3) ILO, 2012.
(4) ILO,2009. 
alcohol and drugs abuse, mobbing, bullying and other forms of violence have also increased. Some studies have shown that stress at work and high decision authority increases the risk of depressive and alcohol and drug-related disorders.

These problems have now been acknowledged as having a significant impact on workers' health and well-being, performance and absenteeism. For the individual worker, it involves considerable consequences in terms of physical and mental health disorders, stigmatization, incapacity to work, risk of job loss, strained or fractured relationships at home, and even disability. Furthermore, the combined effects of smoking, alcohol and drug abuse, nutritional deficiencies, and physical inactivity can also interact with workplace hazards and may increase health risks for workers. Within the enterprise, these problems can also result in disturbed labour relations, increased absenteeism, staff turnover and retraining, reduced motivation, decreased satisfaction and creativity and a poorer public image. These situations have often negative effects on the enterprises' safety, productivity and overall morale. Therefore, they have a considerable impact on productivity.

When such situations affect a group or a population, such as with the present recession in Europe, it becomes a collective problem and has major implications for the well-being of workers, their families and societies as a whole. In 2008 the global unemployment rate was estimated at 6.6 per cent.(5) The economic crisis resulted in an additional 7.8 million young workers facing unemployment, bringing the total to an estimated 81 million, or 13 per cent globally in 2010.(6) This situation has increased work-related stress for those that are still at work, young unemployed workers, and workers in distress from losing their jobs and unable to find new employment. Unemployment can also have serious consequences to the unemployed worker and the family, including stress, depression, violence and even suicide.(7) As an example, in a study published in 2011, researchers found that suicides rose from 2007 to 2009 in nine of the 10 European countries they studied. The upward trend in suicides in Europe began in 2008, when the euro zone entered a recession. The countries "facing the most severe financial reversals of fortune," saw a greater rise in suicides. The most dramatic change was in Greece, where the number of suicides rose to 19 per cent. The study found that for every 1 per cent increase in unemployment, there was an associated 0.8 per cent increase in suicides in people under 65(8).

The related direct and indirect costs are only beginning to be quantified. In 2002, the European Commission reported that the annual cost of work-related stress in the EU15 was EUR 20,000 million(9). In 2005/06 over 70 million days were lost In the United Kingdom due to poor mental health.(10) What is known probably represents only the tip of the iceberg. The overall impact is a significant loss of competitiveness of the enterprise. A report from Safe Work Australia from 2012 found that depression costs Australian employers approximately $\$ 8$ billion Australian dollars (AUD) as a result of sickness absence and presenteeism; from these, job strain and bullying correspond to $\$ 693$ million AUD per year. A prominent finding is that the cost is mostly due to workers showing mild symptoms of depression that take twice as many sick days as those who do not show any symptoms of depression at all. The results further suggest that potentially $\$ 17.84$ billion AUD could be saved in costs to the employer if the mental well-being of the 25 per cent least psychologically healthy workers could be raised to the level of the 25 per cent most psychologically healthy workers through preventive measures(11).

\footnotetext{
(5) ILO, 2010a.

(6) ILO, 2010b.

(7) ILO, 2009; ILO, 2012.

(8) David Stuckler et al, 2011.

(9) EU-OSHA, 2009.

(10) HSE, 2007.

(11) Safe Work Australia, 2012.
} 


\section{THE WORKPLACE CHALLENGE}

In this complex context, the workplace has become an important source of psychosocial risks and poor work-life balance. Consequently, it has also become an ideal venue to address psychosocial risks. Employers should be aware of the negative effects of the psychosocial hazards that may affect workers as a result of overwork and lack of control over their tasks which result in work-related stress and related coping behaviours. The workplace can offer support and allow workers to become more productive without enduring the effects of negative stress. Making employers and workers aware, informed and competent to take care of these new risks, creates a safe and healthy environment, builds a positive and constructive preventive culture in the organization, boosts engagement and effectiveness, protects the health and wellbeing of workers and increases productivity.

For this reason, enterprises should not only focus on an individual response to the problem. A comprehensive approach that breaks away from traditional efforts and moves towards new effective responses is necessary. It is essential to find innovative ways in dealing with the consequences of psychosocial risks and work-related stress in the workplace both with collective and individual measures. The ideal response to stress is to prevent its occurrence. This may be achieved by tackling the core of the problem: the causes. However, as we said before, there is no single cause of stress and the elimination of all psychosocial risks is not always feasible. As multiple psychosocial factors can cause stress, it cannot be assessed and managed in isolation. Both individual and organizational factors which may be contributing to psychosocial risks should be taken into account in order to adapt work to suit workers capabilities and physical and mental health requirements. In addition, it is important to consider both labour and social relations as factors that also have an impact on the well-being of workers and the productivity of the enterprise. Therefore, action should be aimed at eliminating as many workplace causes as possible, so that the action taken reduces and prevents future work-related stress. It is generally agreed that improving the individual's ability to cope with stress can be a valuable complementary strategy as part of the wider, collective and organizational process of combating work-related stress.

The best way to deal with work-related stress is by means of strategies to tackle the psychosocial hazards and risks which are at its source and determine it in the organizational culture and labour relations of the enterprise. A comprehensive OSH management system(12) should ensure improving preventive practices and incorporating health promotion measures as to include psychosocial risks into risk assessment and management measures to effectively manage their impact in the same way as with other OSH risks in the workplace. This implies conducting an occupational health practice with a multi-pronged approach which involves:

- preventing occupational and other work-related diseases, as well as occupational injuries;

— improving working conditions and work organization;

— incorporating psychosocial risks into risk-assessment and management measures, and Implementing collective preventive measures (as done with other workplace hazards and risks) by adapting work organization and working conditions;

— increasing the coping ability of workers;

- building up social support systems for workers within the workplace; and

- assessing the needs of the organization taking into consideration organizational, individual and individual-organizational interactions when evaluating workers' health requirements.

Workers' participation in this process is crucial. Workers should be involved in identifying those psychosocial risks which they feel cause unnecessary stress in their jobs, and in rating them in order to establish priorities for intervention. The assessment should be done in a systematic way and workers should be asked to express their concern about any situation that may be causing stress at work. Once the existence of stress has been recognized and the psychosocial risks at its origin identified, action to deal them at the source should be taken.

(12) Risk management is a problem solving approach to health and safety hazards is an integral part of an enterprise's occupational safety and health $(\mathrm{OSH})$ management system and contributes to the cycle of continuous improvement of work and working conditions 


\section{THE SOLVE PROGRAMME: ILO ACTION IN THE FIELD OF WORK-RELATED STRESS.(13)}

The prevention of work-related stress is an important aspect of the ILO's primary goal on health promotion at the workplace. Close collaboration between management and workers is indispensable in finding solutions for safety and health problems in the workplace with the active participation and involvement of workers and their representatives.

One of the main tasks of the ILO is to support its Constituents and social partners in protecting workers' health and well-being. The ILO developed SOLVE: Integrating bealth promotion into workplace policies (14) with the aim of integrating workplace health promotion into OSH. The SOLVE training programme focuses on the promotion of health and well-being at work through policy design and action to offer an integrated workplace response in addressing the following areas and their interactions:

1. Psychosocial health:

- stress;

- psychological and physical violence;

- economic stressors.

2. Potential addictions and their effects on the workplace:

- tobacco consumption and exposure to second-hand smoke;

- alcohol and drug consumption.

3. Lifestyle habits:

- adequate nutrition;

- exercise or physical activity;

- healthy sleep;

- HIV and AIDS.

SOLVE uses a training-of-trainers methodology. It offers tools for designing a workplace policy, a preventive programme and a plan of action to reduce or eliminate the emerging psychosocial risks associated with the above problems in the workplace with a gender sensitive approach. Participants face real problemsolving situations in the context of a "virtual enterprise". SOLVE also introduces an innovative approach whereby workers' health, safety and well-being become an integral part of organizational development, productivity and competitiveness contributing to the economic sustainability of the enterprise.

The ILO's comparative advantage lies in its experience in using social dialogue in the implementation of successful workplace and community initiatives addressing these problems by means decent work country programmes in member States with the involvement of employers, workers and their representatives, OSH practitioners, governments, policy makers, public services and NGOs. The SOLVE training programme is also part of the curricula of the ILO Training Centre in Turin, Italy.

Providing for mechanisms to address psychosocial risks at work by incorporating preventive and health promotion measures contributes to a more decent and human world of work. The ILO acknowledges that in times of workplace change coping successfully with psychosocial risks at the workplace is essential for protecting the health and well-being of workers while enhancing the productivity of enterprises.

(13) ILO, 2012

(14) http://www.ilo.org/safework/info/instr/WCMS_178438/lang--en/index.htm 